2. Dhawan V, Mohamed A, Fedorak RN. Gastric intramural hematoma: a case report and literature review. Canadian J Gastroenterol 2009; 23: $19-22$

\section{SUDDEN DEATH DUE TO CARDIAC SARCOIDOSIS: A CASE REPORT}

Jessica Vidler, Andrew Kedziora

Forensic and Scientific Services, Queensland Health, Australia

Sarcoidosis is an infiltrative inflammatory disease of unknown aetiology, which can involve multiple organ systems in affected individuals. The disease is characterised by the histologic hallmark of non-caseating epithelioid granulomas, for which other causes of granulomatous infiltrates have been excluded. Cardiac involvement occurs in up to one-third of cases and indicates an adverse prognosis, with cardiac sarcoidosis being responsible for approximately one-half to two-thirds of sarcoid-related deaths. Clinical cardiac manifestations preceding death, such as arrhythmia, heart block or heart failure, only occur in approximately $5 \%$ of people with cardiac sarcoidosis, and therefore a diagnosis of cardiac sarcoidosis is often not made prior to death. We present a case of an older age Caucasian male who was unexpectedly found dead in his home. The cause of death in this case was cardiac sarcoidosis. Through this case we highlight the macroscopic and microscopic features of cardiac sarcoidosis and present a rationale for the importance of internal autopsy in cases that may otherwise be assigned a cause of death based on patient history, CT scan findings, and an external-only post-mortem examination

\section{RAPID EXOME SEQUENCING AND ADJUNCT RNA STUDIES CONFIRM PATHOGENICITY OF A NOVEL HOMOZYGOUS ASNS SPLICING VARIANT IN A CRITICALLY ILL NEONATE}

Lauren S. Akesson ${ }^{1,2,3}$, Adam Bournazos ${ }^{4,5}$, Andrew Fennell ${ }^{3,6}$, Emma I. Krzesinski ${ }^{3,6}$, Kenneth Tan ${ }^{6,7}$, Amanda Springer ${ }^{3,6}$, Katherine Rose ${ }^{3,6}$, Ilias Goranitis ${ }^{2,8,9}$, David Francis ${ }^{1}$, Crystle Lee $^{1}$, John Christodoulou ${ }^{1,2,8,9}$, Sebastian Lunke ${ }^{1,2,9}$, Zornitza Stark $^{1,2,9}$, Matthew F. Hunter ${ }^{3,6}$, Sandra Cooper ${ }^{4,5,10}$ ${ }^{1}$ Victorian Clinical Genetics Services, Melbourne, Vic, Australia; ${ }^{2}$ University Of Melbourne, Melbourne, Vic, Australia; ${ }^{3}$ Monash Genetics, Monash Health, Melbourne, Vic, Australia; ${ }^{4}$ Kids Neuroscience Centre, Children's Hospital at Westmead, Sydney, NSW, Australia; ${ }^{5}$ University of Sydney, Sydney, NSW, Australia; ${ }^{6}$ Monash University, Melbourne, Vic, Australia; ${ }^{7}$ Monash Newborn, Monash Health, Melbourne, Vic, Australia; ${ }^{8}$ Murdoch Children's Research Institute, Melbourne, Vic, Australia; ${ }^{9}$ Australian Genomics Health Alliance, Australia; and ${ }^{10}$ Children's Medical Research Institute, Sydney, NSW, Australia

Rapid genomic diagnosis programs are transforming rare disease diagnosis in paediatric acute care. Determining the pathogenicity of variants of uncertain significance within clinically relevant timeframes remains challenging. A ventilated newborn with cerebellar hypoplasia underwent rapid exome sequencing, identifying a novel homozygous ASNS splice-site variant (NM_133436.3:c.1476+1G>A) of uncertain significance in 75.5 hours. Rapid ASNS splicing studies using blood-derived mRNA from the family trio confirmed a consistent pattern of abnormal splicing induced by the variant (cryptic $5^{\prime}$ splice-site or exon 12 skipping) with absence of normal ASNS splicing in the proband. Splicing studies were reported within 10 days and led to reclassification of c. $1476+1 \mathrm{G}>\mathrm{A}$ as pathogenic at age 27 days, diagnosing asparagine synthetase deficiency (MIM 615574). Intensive care was redirected towards palliation. Time from initial exome sequencing report to variant reclassification was 22 days. Cost analysis based on hospitalisation length of stay for the proband and his similarly affected deceased sibling demonstrated that early diagnosis led to a reduction in hospitalisation costs by AU $\$ 17,800$. We highlight diagnostic benefits of adjunct RNA testing to confirm pathogenicity of splicing variants identified via rapid genomic testing pipelines for precision and preventative medicine.

\section{MEASURING THE QUALITY OF PATHOLOGY REPORTS}

Habib Taouk $^{1,2}$, Ma. Juliana Leon R. ${ }^{1}$, Ebony Richardson ${ }^{1,2}$, Sharon Young ${ }^{2}$, Leslie Burnett

${ }^{1}$ Kinghorn Centre for Clinical Genomics, Garvan Institute of Medical Research, Darlinghurst, Sydney, NSW, Australia; ${ }^{2}$ Genome.One, Australian Clinical Laboratories, Darlinghurst, Sydney NSW, Australia; ${ }^{3}$ St Vincent's Clinical School, UNSW Sydney, Darlinghurst, Sydney NSW, Australia; and ${ }^{4}$ Sydney Medical School, University of Sydney, Sydney, NSW, Australia

Pathology has traditionally used process-based tools to assure safety and quality. These include risk-based standards focussing on staffing competencies, equipment maintenance, procedure standardisation, and use of a quality management system (QMS). Within the QMS, quality is systematically monitored through quality control, quality assurance (QA) programs and audits.

We have previously shown that the major opportunities for error lie at boundaries between the pathology laboratory and the rest of the health system. Our previous work developed pre-analytical QA tools. We now describe the development of a post-analytical QA tool.

Our new tool allows quantitative measure of the quality of the final pathology report. Using genetic pathology as an example, we measured the quality of every final pathology report produced by a laboratory over a $2 \frac{1}{2}$ year period. Statistical measures calculated the likelihood of an error being present, whether such errors were major or minor, and whether the rates of errors detected were statistically stable. Applying this tool to the laboratory resulted in a 2- to 5-fold reduction in frequency of errors and quantitative improvement in quality of pathology reports. Although our tool has been piloted in genetic pathology, it is readily applicable to all pathology disciplines.

\section{PETER MAC EXPERIENCE WITH COMPREHENSIVE GENOMIC PROFILING - THE NEW STANDARD OF CARE IN TISSUE PATHOLOGY?}

Andrew Fellowes $^{1}$, Christopher McEvoy ${ }^{1}$, Huiling $\mathrm{Xu}^{1}$, Anna Tanska ${ }^{1}$, Ain Roesley ${ }^{2}$, David Choong ${ }^{1}$, Roxane Legaie ${ }^{1}$, Stephen B. Fox ${ }^{1}$

${ }^{1}$ Department of Pathology, Peter MacCallum Cancer Centre, Parkville, Melbourne, Australia; and ${ }^{2}$ Victorian Clinical 